\title{
FINITE ABELIAN ACTIONS ON SURFACES
}

\author{
by S. A. JASSIM
}

(Received 6 December, 1991)

Let $G$ be a finite abelian group of rank $m, M$ an oriented compact connected surface, and $F(G, M)$ the set of all orientation preserving free $G$-actions on $M$. Two actions $\Phi_{1}$, $\Phi_{2} \in F(G, M)$ are equivalent if there exists an orientation preserving homeomorphism $h$ of $M$ such that

$$
h \Phi_{1}(f) h^{-1}=\Phi_{2}(f) \text { for all } f \in G .
$$

If $F(G, M)$ is nonempty then there is an oriented surface $N \equiv M / G$ such that for each $\Phi \in F(G, M), M / \Phi$ is homeomorphic to $N$. The classification of the elements of $F(G, M)$ has been the subject of much research work (see e.g. [2], [3], [4], [5], [7], [8], [9], [10], and [11]). A. Edmonds, in [2], showed that the bordism invariant of such actions defines an injective function $\mathbf{B}: F(G, M) / \sim \rightarrow H_{2}(G)$. If $m \leq \operatorname{genus}(N)$, then $\mathbf{B}$ is a bijection (see [2]). However the characterisation of the set $\operatorname{Im}(B)$ in the remaining cases, i.e. when $\operatorname{genus}(N)<m \leq 2$ genus $(N)$, has remained open. It is only known that $0 \notin \operatorname{Im}(\mathbf{B})$. The aim of this paper is to solve this problem when $G=\left(\mathbf{Z}_{p}\right)^{m}$ and $p$ is a prime number. The main ingredient of the solution, given in Section 2 , is a technical result relating the determinant of a matrix to a recursively defined polynomial in the symplectic products of its rows. Slight modifications of the arguments, in Section 2, lead to a solution of the problem for general primary abelian groups. For nonabelian groups $G, \mathrm{C}$. Livingston [7] and B. Zimmerman [11] studied the relation between $\mathrm{H}_{2}(G)$ and the stable equivalence classes of $G$-actions on surfaces.

In Section 1, we reduce the problem of classifying free $G$-actions on surfaces to that of classifying free actions of the primary decompositions of $G$, and introduce the symplectic invariant on matrices through which the bordism invariant factors. We also give a simple constructive proof of the injectivity of the symplectic invariant which depends on the original Witt's theorem for symplectic vector spaces over finite fields. Throughout all homeomorphisms are orientation preserving, and all homology groups are taken with integral coefficients. If $R$ is an equivalence relation on a set $X$ then $X / R$ is written as $X^{*}$.

1. Let $G$ be a finite abelian group and $M$ an oriented compact connected surface. For any $\Phi \in F(G, M)$, the map $p_{\Phi}: M \rightarrow M / \Phi$ is a regular $G$-covering. Let $C(G, N)$ be the set of all regular $G$-covering projections over $N$. Two regular $G$-coverings $p_{1}, p_{2}: M \rightarrow N$ are equivalent if there exist homeomorphisms $F$ of $M$, and $f$ of $N$ such that $F$ is a $G$-map and $f p_{1}=p_{2} F$. If $f=I_{N}$ then $p_{1}$ and $p_{2}$ are isomorphic. Up to isomorphism, each element $p \in C(G, N)$ is uniquely determined by an epimorphism $\rho_{p}: H_{1}(N) \rightarrow G$. For any two groups $K$ and $L$, let $E(K, L)$ be the set of all epimorphisms from $K$ to $L$. If $g=\operatorname{genus}(N)$, then $H_{1}(N) \approx \mathbf{Z}^{2 g}$ and the intersection number defines a symplectic product on $H_{1}(N)$. The set $\mathbf{B}$ of the homology classes of the simple closed curves $\left\{a_{1}, b_{1}, \ldots, a_{g}, b_{g}\right\}$, shown in Figure 1 , forms a symplectic basis for $H_{1}(N)$, which is fixed throughout this paper. Two epimorphisms $\rho_{1}, \rho_{2} \in E\left(H_{1}(N), G\right)$ are said to be equivalent if there exists a symplectic automorphism $\alpha$ of $H_{1}(N)$ such that $\rho_{1} \alpha=\rho_{2}$. The sets

Glasgow Math. J. 35 (1993) 225-234. 


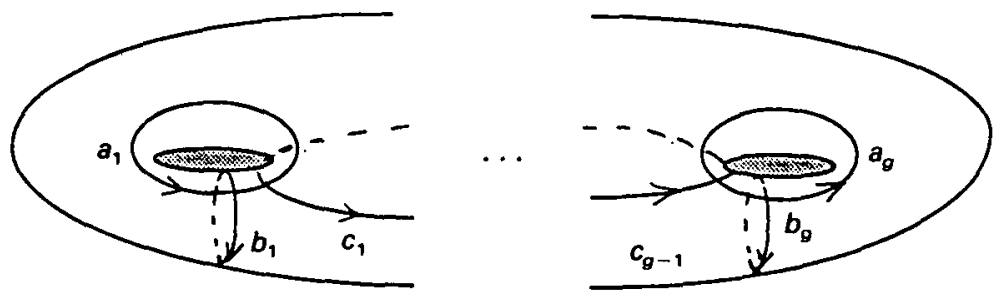

Figure 1.

$F^{*}(G, M), C^{*}(G, N)$, and $E^{*}\left(H_{1}(N), G\right)$ are in bijection with each other (see e.g. [2], or [5]). A symplectic automorphism of $\mathbf{Z}^{2 g}$ is one which preserves the symplectic inner product. A symplectic automorphism of $\mathbf{Z}^{2 g}$ is said to be elementary if and only if

$$
\forall x \in \mathbf{B}, \exists y \in \mathbf{B} \cup\{0\} \text { such that } \alpha(y)=y \text { and } \alpha(x)=x \pm y .
$$

The group $\mathrm{Sp}_{2 g}(\mathbf{Z})$ of symplectic automorphisms of $\mathbf{Z}^{2 g}$ is generated by $3 g-1$ elementary automorphisms which are induced by the "Dehn twists" around the simple closed curves $\left\{a_{1}, b_{1}, \ldots, a_{g}, b_{g}, c_{1}, \ldots, c_{g-1}\right\}$, shown in Figure 1. This is the main factor in the proof of the following proposition.

1.1. Proposition. If $G_{1}$ and $G_{2}$ are finite abelian groups of relatively prime order and $N$ is a closed surface, then the map

$$
E^{*}\left(H_{1}(N), G_{1} \oplus G_{2}\right) \rightarrow E^{*}\left(H_{1}(N), G_{1}\right) \times E^{*}\left(H_{1}(N), G_{2}\right):[\rho] \rightarrow\left(\left[\tau_{1} \rho\right],\left[\tau_{2} \rho\right]\right)
$$

is a bijection, where $\tau_{i}: G_{1} \oplus G_{2} \rightarrow G_{i}$ is the natural projection onto the $i$-th coordinate. (See [5].)

1.2. Corollary. If $G=\bigoplus G_{p_{i}}, i=1, \ldots, n$, is the primary decomposition of $G$ and $N$ is a closed surface, then there are bijections

$$
\begin{aligned}
& \Im: C^{*}(G, N) \rightarrow \prod_{i=1}^{m} C^{*}\left(G_{p_{i}}, N\right), \\
& \Im: F^{*}(G, M) \rightarrow \prod_{i=1}^{m} F^{*}\left(G_{p_{i}}, M_{i}\right),
\end{aligned}
$$

where $M_{i}$ is a homeomorphic image of a close surface of genus

$$
1+\left|G_{p_{i}}\right|(\operatorname{genus}(M)-1) /|G| \text {. }
$$

Let $p$ be a prime number, and $G=\bigoplus \mathbf{Z}_{p^{k_{i}}}$, where $k_{1} \leq \ldots \leq k_{m}$. We fix as a basis for $G$ the set $\left\{e_{1}, \ldots, e_{m}\right\}$, where $e_{i}$ is the vector with 1 in the $i$ th position but zero elsewhere. For any $\Phi \in F(G, M)$ (equivalently $p \in C(G, N)$ ), let $B_{\Phi}$ be the $m \times 2 g$ integral matrix representing

$$
\rho_{p}: H_{1}(G) \rightarrow G
$$

with respect to the fixed bases. $B_{\Phi}$ has row rank $m$ and its $i$ th row consists of integers modulo $p^{k_{i}}$. Let $M(g, G)$ be the set of all such matrices. Two matrices $B_{1}, B_{2} \in M(g, G)$ are said to be equivalent if for some $S \in \mathrm{Sp}_{2 g}(\mathbf{Z}), B_{1}=B_{2} S$. Each of $F^{*}(G, M)$ and $C^{*}(G, N)$ is in bijection with $M^{*}(g, G)$. 
The column operations that are needed to prove symplectic equivalences can be achieved by a small list of matrices in $\operatorname{Sp}_{2 g}(\mathbf{Z})$. The list contains, for $1 \leq i, j \leq g$, the following matrices which are obtained from $I_{2 g}$ by the indicated operations, as well as their inverses:

$\alpha_{i}$ : adding the $i$ th column to the $(i+g)$ th column,

$\beta_{i}$ : adding the $(i+g)$ th column to the $i$ th column,

$\delta_{i j}$ : adding the $i$ th column to the $j$ th column, and subtracting the $(j+g)$ th column from the $(i+g)$ th column,

$\xi_{i j}$ : adding the $i$ th column to the $(j+g)$ th column, and the $j$ th column to the $(i+g)$ th column,

$\sigma_{i j}$ : interchanging the $i$ th column with the $j$ th column, and the $(i+g)$ th column with the $(j+g)$ th column,

$\tau_{i j}$ : interchanging the $i$ th column with the $(j+g)$ th column, the $j$ th column with the $(i+g)$ th column (if $i \neq j$ ), and then multiplying the $(i+g)$ th column and the $(j+g)$ th column by -1 , and

$\lambda_{i j}:\left(\xi_{i j}\right)^{t}$.

1.3. Lemma. If $1 \leq m<2 g$ and $A \in M(g, G)$, then there is an integer $i, i \leq i \leq 2 g$, such that $A$ is equivalent to a matrix $B$ whose $i$-th column is a zero one.

Proof. Since $\operatorname{rank}(A)=m<2 g$, then, up to symplectic equivalence, there is a column of $A$ which is a linear combination of the previous ones. Let $\mathbf{c}_{i}$ be the first such column and suppose that

$$
\mathbf{c}_{i}=a_{1} \mathbf{c}_{1}+\ldots+a_{i-1} \mathbf{c}_{i-1} .
$$

If $i \leq g$, then $B=A\left(\delta_{1 i}\right)^{-a_{1}} \ldots\left(\delta_{i-1}\right)^{-a_{i-1}}$ is the required matrix. If $i=g+1$, then $B=A\left(\alpha_{1}\right)^{-a_{1}} \tau_{11}\left(\delta_{21}\right)^{-a_{2}} \ldots\left(\delta_{g 1}\right)^{-a_{8}}$ is the required matrix. For $i>g+1$, let $k=i-g$ and $d=a_{k}-a_{1} a_{g+1}-\ldots-a_{k-1} a_{g+k-1}$. Then

$$
B=A\left(\delta_{1 k}\right)^{a_{g+1}} \ldots\left(\delta_{k-1 k}\right)^{a_{i-1}}\left(\xi_{1 k}\right)^{-a_{1}} \ldots\left(\xi_{k-1 k}\right)^{-a_{k-1}}\left(\alpha_{k}\right)^{-d}\left(\xi_{k+1 k}\right)^{-a_{k+1}} \ldots\left(\xi_{g k}\right)^{-a_{g}}
$$

is the required matrix.

1.4. Definition. Let $B \in M(g, G)$ be any matrix. For any two integers $i$ and $j$ with $1 \leq i<j \leq m$, let

$$
B_{i j}=\left\langle r_{i}(B), r_{j}(B)\right\rangle \bmod p^{k_{i}},
$$

where $\left\langle r_{i}(B), r_{j}(B)\right\rangle$ is the symplectic product of the $i$ th and $j$ th rows of $B$. The symplectic invariant is the function

$$
\xi: M(g, G) \rightarrow H_{2}(G)
$$

defined for each matrix $B \in M(g, G)$ as:

$$
\xi(B)=\left(B_{12}, \ldots, B_{1 m}, B_{23}, \ldots, B_{2 m}, \ldots, B_{m-1 m}\right) .
$$

Note that, $H_{2}(G ; \mathbf{Z})=\left(\mathbf{Z}_{p^{k_{1}}}\right)^{m-1} \oplus\left(\mathbf{Z}_{p^{k_{2}}}\right)^{m-2} \oplus \ldots \oplus \mathbf{Z}_{p^{k_{m-1}}}$. 
The domain of $\xi$ can be extended to the set $M_{m, g}$ of all $m \times 2 g$ integral matrices in the obvious way, and sometimes we will refer to this extension. It is obvious that equivalent matrices in $M(g, G)$ have the same symplectic invariant and hence $\xi$ is a function on $M^{*}(g, H)$. Up to an isomorphism of $G$, related to the choice of basis for $G$, the Bordism invariant

$$
\mathbf{B}: F(G, M)^{*} \rightarrow H_{2}(G),
$$

defined by Edmonds in [2], factors through $\xi$. By proving a generalisation of Witt's theorem to symplectic spaces over local rings, Edmonds showed that $\mathbf{B}$ is an injection. In fact, Edmonds proved that $\xi$ is injective. However, we shall now provide a simple, and somehow constructive, proof of the injectivity of $\xi$ in which only the original Witt's theorem for symplectic vector spaces over finite fields is needed. We first prove a lemma which results in the reduction of the problem in the case of general $p$-groups into that of groups of the form $\left(\mathbf{Z}_{p^{k}}\right)^{r}$.

For any $p$-group $G=\bigoplus\left(\mathbf{Z}_{p^{k_{i}}}\right)$, with $k_{1} \leq \ldots \leq k_{r}$, let $\mathbf{G}=\left(\mathbf{Z}_{p^{k_{r}}}\right)^{r}$. For any natural number $g$, the modulo projection $\rho: \mathbf{G} \rightarrow G$ induces a function $\rho_{g}: M(g, \mathbf{G}) \rightarrow M(g, G)$.

1.5. Lemma. Let $1 \leq h<k, G=\mathbf{Z}_{p^{h}} \oplus\left(\mathbf{Z}_{p^{k}}\right)^{m}, A \in M(g, G)$ any matrix with $1 \leq m<$ $2 g$, and let $\xi(A)=\left(\xi_{12}, \ldots, \xi_{m m+1}\right)$. If $\sigma=\left(s_{12}, \ldots, s_{1 m+1}\right)$ is any sequence of numbers in $\mathbf{Z}_{p^{k}}$ such that $s_{1 i} \equiv \xi_{1 i}\left(\bmod p^{h}\right), i=1, \ldots, m+1$, then there exists a matrix $\mathbf{A} \in$ $M(g, \mathbf{G})$ such that $\rho_{g}(\mathbf{A})$ is equivalent to $A$, and $\xi(\mathbf{A})=\left(s_{12}, \ldots, s_{1 m+1}, \xi_{23}, \ldots, \xi_{m m+1}\right)$.

Consequently, if $G$ is any abelian $p$-group and $A, B \in M(g, G)$ are two matrices such that $\xi(A)=\xi(B)$, then there exists a matrix $\mathbf{B} \in M(g, \mathbf{G})$ such that $\rho_{g}(\mathbf{B})$ is equivalent to $B$, and, as matrices in $M(g, \mathbf{G}), \xi(A)=\xi(\mathbf{B})$.

Proof. The proof is by induction on $m$.

If $m=1$, then by definition there is a positive integer $i$ such that, up to symplectic equivalence, $2 g>i>g$ and $a_{2 i}=1$. If $s_{12}-\xi_{12}=t p^{h}$, as integers, then the matrix $\mathbf{A} \in M(g, \mathbf{G})$, obtained by replacing $a_{1 i-g}$ with $a_{1 i-g}+t p^{h}$, is the required matrix.

Suppose that the lemma is true for $m=r$ and let $G=\mathbf{Z}_{p^{h}} \oplus\left(\mathbf{Z}_{p^{k}}\right)^{r+1}$. Let $A^{\prime}$ be the submatrix of $A$ obtained by deleting the 2 nd row, and consider the subsequence $\sigma^{\prime}=\left(s_{13}, \ldots, s_{1 m+1}\right)$. Let $\mathbf{A}^{\prime} \in M\left(g, \mathbf{G} / \mathbf{Z}_{p^{k}}\right)$ be the matrix that satisfies the conditions above, and obtained by the induction hypothesis. Let $B$ be the matrix obtained from $\mathbf{A}^{\prime}$ by inserting the 2 nd row of $A$ between its 1 st and 2 nd row. Obviously, $B$ is of rank $r+2$. Hence, there exists a positive integer $i$ such that, up to symplectic equivalence, $2 g>i>g$, $b_{2 i}=1$, and all the entries of $B$ below $b_{2 i}$ are zeros. Again, if $s_{12}-\xi_{12}=t p^{h}$, as integers, then the matrix $\mathbf{A} \in M(g, \mathbf{G})$ obtained from $B$ by replacing $b_{1 i-g}$ with $b_{1 i-g}+t p^{h}$, is the required matrix.

1.6. Proposition. The symplectic invariant is injective on $M^{*}(g, G)$ (i.e. if $\xi(A)=$ $\xi(B)$ then $A$ is equivalent to $B$ ).

Proof. By 1.5 , one can assume that $G$ is a free $\mathbf{Z}_{p^{k}}$-module. The proof is divided into 2 cases.

Case (1): $m=2 g$. Let $A^{-1}$ be the inverse of the matrix $A$ considered as a $\mathbf{Z}_{p^{k}}$ matrix, and consider the $2 \mathrm{~g} \times 2 \mathrm{~g}$ matrix $Q=A^{-1} B$. Let

$$
J_{2 g}=\left[\begin{array}{cc}
0 & -I_{g} \\
I_{g} & 0
\end{array}\right] .
$$


Since $\xi(A)=\xi(B)$, then $A J_{2 g} A^{\mathrm{t}}=B J_{2 g} B^{\mathrm{t}}$. Hence

$$
Q J_{2 g} Q^{\mathrm{t}}=A^{-1} B J_{2 g} B^{\mathrm{t}}\left(A^{\mathrm{t}}\right)^{-1}=A^{-1} A J_{2 g} A^{\mathrm{t}}\left(A^{\mathrm{t}}\right)^{-1}=J_{2 g},
$$

i.e. $Q \in \mathrm{Sp}_{2 g}\left(\mathbf{Z}_{p^{k}}\right)$. The fact that $A Q=B$ proves the result in this case.

Case (2): $m<2 g$. Since $A$ is of rank $m<2 g$ then there is a column of $A$, say the $i$ th, which can be assumed, by 1.3 , to be a zero column. Let $A^{\prime}$ be the matrix whose 1 st row is the unit vector $\mathbf{e}_{i}$ in $\left(\mathbf{Z}_{p}\right)^{2 g}$, and for $j>1$ its $j$ th row is the $(j-1)$ th row of $A$. Let $\mathbf{A}^{\prime}$ and $\mathbf{B}$ be the $\mathbf{Z}_{p}$ matrices obtained from $A^{\prime}$ and $B$, respectively, by reducing all entries $\bmod p$. Now, $\mathbf{A}^{\prime}$ and $\mathbf{B}$ are of rank $m+1$ and $m$, respectively. Then by the original Witt's theorem for symplectic spaces over $\mathbf{Z}_{p}$, there is a vector $\mathbf{v} \in\left(\mathbf{Z}_{p}\right)^{2 g}$ which is linearly independent of the rows of $\mathbf{B}$ and such that

$$
\left\langle\mathbf{v}, r_{j}(\mathbf{B})\right\rangle \equiv\left\langle\mathbf{e}_{i}, r_{j+1}\left(\mathbf{A}^{\prime}\right)\right\rangle(\bmod p), \quad \text { for } j=1, \ldots, m .
$$

Let $B^{\prime}$ be the $(m+1) \times 2 g$ matrix whose 1 st row is the vector $v \in\left(\mathbf{Z}_{p}\right)^{2 g}$, w.r.t. the standard basis, and for $j>1$ its $j$ th row is the $(j-1)$ th row of $B$. Now, $A^{\prime}$ and $B^{\prime}$ are two $(m+1) \times 2 g$ matrices in $M\left(g, \mathbf{Z}_{p} \oplus G\right)$ such that $\xi\left(A^{\prime}\right)=\xi\left(B^{\prime}\right)$ in $H_{2}\left(\mathbf{Z}_{p} \oplus G\right)$. Repeating this process $2 g-m$ times produces two matrices $A^{\prime \prime}$ and $B^{\prime \prime}$ in $M\left(g,\left(\mathbf{Z}_{p}\right)^{2 g-m} \oplus G\right)$, whose last $m$ rows are those of $A$ and $B$, respectively, $\xi\left(A^{\prime \prime}\right)=$ $\xi\left(B^{\prime \prime}\right)$ in $H_{2}\left(\left(Z_{p}\right)^{2 g-m} \oplus G\right)$. By case (1), and Lemma 1.5 , there exists a symplectic $2 g \times 2 g$ matrix $S$ such that $A^{\prime \prime} S=B^{\prime \prime}$. Clearly $A S=B$, and the proof is complete.

1.7. Proposition. If $1 \leq m \leq g$, then $\xi$ is surjective.

Proof. Let $\mathrm{v}=\left(v_{12}, \ldots, v_{1 m}, v_{23}, \ldots, v_{m-1 m}\right) \in H_{2}(G)$ be any element. If

$$
A=\left[\begin{array}{cccccccccccccc}
1 & 0 & \ldots & 0 & 0 & \ldots & 0 & 0 & v_{12} & \ldots & v_{1 m} & 0 & \ldots & 0 \\
0 & 1 & \ldots & 0 & 0 & \ldots & 0 & 0 & 0 & \ldots & v_{2 m} & 0 & \ldots & 0 \\
& & \vdots & & & \vdots & & & & \vdots & & & \vdots & \\
0 & 0 & \ldots & 1 & 0 & \ldots & 0 & 0 & 0 & \ldots & v_{m-1 m} & 0 & \ldots & 0 \\
0 & 0 & \ldots & 0 & 1 & \ldots & 0 & 0 & 0 & \ldots & 0 & 0 & \ldots & 0
\end{array}\right]
$$

then, $A \in M(g, G)$ and $\xi(A)=\mathbf{v}$.

1.8. COROLLARY. If $M$ is a closed surface of genus $g^{*}$ and if $m$ is an integer such that $1 \leq m \leq 1+\left(g^{*}-1\right) /|G|$, then there are $p^{K}$ equivalence classes of free $G$-actions on $M$, where

$$
K=\sum_{i=1}^{m}(m-i) k_{i}
$$

2. This section is devoted to the characterisation of the vectors in $\operatorname{Im}(\xi)$, when $g<m \leq 2 g$. This completes the solution of the classification of free actions of elementary abelian groups $G$ on closed surfaces $M$. However, analogous results can be obtained for primary abelian groups by slight modification of the statements and the arguments used. Throughout, all skew-symmetric matrices are assumed to have zero diagonals. First, a technical result, relating the determinant of a $2 n \times 2 n$ matrix to a recursively defined polynomial function $d$ in the symplectic inner products of its rows, is proved. This yields a condition which must be satisfied by any element in $H_{2}(G)$ in order to be in $\operatorname{Im}(\xi)$ when 
$m=2 g$. We then prove that for $g<m<2 g$ one of a number of such conditions, in the $d$ function of some $2(m-g) \times 2(m-g)$ matrices, must be satisfied by vectors in $\mathrm{H}_{2}(G)$ in order to be $\operatorname{Im}(\xi)$.

2.1. Definition. Let $n$ be a natural number, $\mathbf{v} \in \mathbf{Z}^{n(n-1) / 2}$, and let $I=\left(i_{1}, \ldots, i_{t}\right)$ be an increasing sequence of natural numbers not exceeding $n$. By $\mathbf{v}^{\prime}$ we mean the subvector of $\mathbf{v}$ obtained by deleting all coordinates in the set

$$
\bigcup_{j=1}^{t}\left\{\left\{v_{i_{i} i_{j}+1}, \ldots, v_{i_{j} n}\right\} \cup\left\{v_{k i_{j}}: 1 \leq k<i_{j}\right\}\right\} .
$$

If $I=(1, i)$, then we simply write $\mathbf{v}^{i}$.

For even numbers $n$, let

$$
d: \mathbf{Z}^{n(n-1) / 2} \rightarrow \mathbf{Z}
$$

be the function defined inductively on $n$ as follows:

$$
d\left(\left(v_{12}\right)\right)=v_{12}
$$

and, for $n>2$,

$$
d(v)=\sum_{i=2}^{n}(-1)^{i} v_{1 i} d\left(v^{i}\right) .
$$

If $S$ is a skew-symmetric $2 n \times 2 n$ matrix, then we define

$$
d(S)=d\left(\left(s_{12}, s_{13}, \ldots, s_{12 n}, s_{23}, \ldots, s_{22 n}, \ldots, s_{2 n-12 n}\right)\right) .
$$

If $I=\left\{i_{1}, \ldots, i_{t}\right\}$ and $J=\left\{j_{1}, \ldots, j_{t}\right\}$ are sets of distinct natural numbers not exceeding $m$, and $A$ is an $m \times m$ matrix, then $A(I ; J)$ denotes the submatrix of $A$ formed by deleting all rows indexed by $I$ and all columns indexed by $J$. If $I=J$ then we simply write $A(I ;)$. For integers $r, t$ and $n$ with $1<r, t \leqslant 2 n$, let $n(r, t)=\{\{2, \ldots, 2 n\}-\{r, t\}\}$, and

$$
r[t]= \begin{cases}t+r & \text { if } t>r, \\ t+r-1 & \text { if } t<r .\end{cases}
$$

Using these notations, we find that

$$
d(S)=\sum_{k=2}^{2 n}(-1)^{k} s_{1 k} d(S(1, k ;)) .
$$

Moreover, it is not difficult to see that $d(S)$ can be expanded recursively in terms of any row or column. In fact, if $1<r \leqslant 2 n$ then

$$
d(S)=\sum_{r \neq k=1}^{2 n}(-1)^{r[k]} s_{r k} d(S(r, k ;)),
$$

where for $r>k, s_{r k}=-s_{k r}$. For any square matrix $A, D(A)$ denotes the determinant of $A$.

2.2. Lemma. Let $n \geq 2$ be an integer, $S$ a $2 n \times 2 n$ skew-symmetric matrix of integers, and $i$ and $j$ integers such that $2 \leq i \leq j \leq 2 n$. Then

(1) $D(S(1, i ; 1, j))=d(S(1, i ;)) \cdot d(S(1, j ;))$, and

(2) $D(S)=(d(S))^{2}$. 
Proof. The proof is done by induction on $n$.

Base case: $n=2$. (1) If $i^{\prime}=\min \{\{2,3,4\}-\{j\}\}, j^{\prime}=\max \{\{2,3,4\}-\{j\}\}, i^{\prime \prime}=$ $\min \{\{2,3,4\}-\{i\}\}$, and $j^{\prime \prime}=\max \{\{2,3,4\}-\{i\}\}$, then

$$
D(S(1, i ; 1, j))=s_{i} \cdot s_{j} \cdot s_{i^{\prime \prime} j^{\prime \prime}}=d(S(1, j ;)) \cdot d(S(1, i ;)) .
$$

(2) This part follows from the relation

$$
D(S)=\left(s_{12} s_{34}-s_{13} s_{24}+s_{14} s_{23}\right)^{2} .
$$

Induction hypothesis. Assume that both (1) and (2) are true for all $m<n$, and let $S$ be a $2 n \times 2 n$ matrix.

(1) The case $i=j$ follows from the fact that $S(1, i$;) is skew-symmetric and the induction hypothesis. Thus we assume that $i<j$.

Expressing $d(S(1, i ;))$ in terms of its $(j-2)$ th row and $d(S(1, j ;))$ in terms of its $(i-1)$ th row yields:

$$
\begin{aligned}
d(S(1, i ;)) & d(S(1, j ;)) \\
& =\left(\sum_{k \in n(i, j)}(-1)^{(j-2)[k]} s_{j k} d(S(1, i, j, k ;))\right) \cdot\left(\sum_{h \in n(i, j)}(-1)^{(i-1)[h]} s_{i h} d(S(1, i, j, h ;))\right) \\
& =\sum_{k \in n(i, j)} \sum_{h \in n(i, j)}(-1)^{(j-2)[k]+(i-1)[h]} s_{j k} s_{i h} d((S(1, i, j, k ;))) d((S(1, i, j, h ;))) .
\end{aligned}
$$

But $S(1, i, j, k ;)=S(i, j ;)\left(1, k^{\prime} ;\right)$ and $S(1, i, j, h ;)=S(i, j ;)\left(1, h^{\prime} ;\right)$, for some $k^{\prime}$ and $h^{\prime}$. Since $S(i, j ;)$ is a $2(n-1) \times 2(n-1)$ skew-symmetric matrix, then

$$
d(S(1, i, j, k ;)) . d(S(1, i, j, h ;))=D\left(S(i, j ;)\left(1, k^{\prime} ; 1, h^{\prime}\right)\right)=D(S(1, i, j, k ; 1, i, j, h)) .
$$

Therefore

$$
d(S(1, i ;)) d(S(1, j ;))=\sum_{k \in n(i, j)} \sum_{h \in n(i, j)}(-1)^{(j-2)[k]+(i-1)|h|} s_{j k} s_{i h} D((S(1, i, j, k ; 1, i, j, h))) .
$$

The last expression is $D(S(1, i ; 1, j ;))$ when expanded along its $(i-1)$ th column and each resulting minor along its $(j-2)$ th row. Note that here the cofactor of $s_{j i}$ is skewsymmetric of odd size and hence it disappears from the expansion of $D(S(1, i ; 1, j ;))$. The result follows by induction.

(2) By definition

$$
\begin{aligned}
(d(S))^{2} & =\left(\sum_{k=2}^{2 n}(-1)^{k} s_{1 k} d(S(1, k ;))\right)^{2} \\
& =\sum_{k=2}^{2 n} \sum_{h=2}^{2 n}(-1)^{k+h} s_{1 k} s_{1 h} d(S(1, k ;)) d(S(1, h ;)) \\
& =\sum_{k=2}^{2 n} \sum_{h=2}^{2 n}(-1)^{k+h} s_{1 k} s_{1 h} D((S(1, k ; 1, h)) .
\end{aligned}
$$

But the last expression is $D(S)$, when expanded along its 1st row and then each minor along its 1st column.

It was pointed out, by the referee, that the $d$ function is closely related to the pfaffian polynomial. 
2.3. Corollary. If $A$ is a $2 g \times 2 g$ matrix of integers, then

$$
(D(A))^{2}=(d(\xi(A)))^{2} .
$$

Here $\xi$ refers to the extended version.

Proof. This follows from the fact that

$$
A J_{2 g} A^{\prime}=S,
$$

where $S=\left[s_{i j}\right]$ is the skew-symmetric $2 g \times 2 g$ matrix such that $s_{i j}$ is the symplectic product of the $i$ th and $j$ th rows of $A$.

2.4. Proposition. Let $k$ and $g$ be integers with $1 \leq k<g$. If $A$ is $a(g+k) \times 2 g$ matrix over $\mathbf{Z}_{p}$ of rank $m=g+k$, then up to symplectic equivalence there exists an increasing sequence of natural numbers $I=\left(i_{1}, \ldots, i_{g-k}\right)$ not exceeding $m$, such that:

(1) the symplectic products of the rows of the submatrix $A(I ; \varnothing)$ are equal to that of the corresponding rows of $A$, and

(2) deleting all the pairs of columns indexed by $j$ and $g+j$, for $1 \leq j \leq g-k$, yields a $2 k \times 2 k$ matrix of rank $2 k$.

Proof. Lemma 1.3 shows that, up to symplectic equivalence, $A$ has a zero column. Multiplication on the right by $\sigma_{i j}$ or $\tau_{i j}$, if necessary, ensures that the $(g+1)$ th column of $A$ is a zero column. Let $A^{\prime}$ be the submatrix of $A$, obtained by deleting the 1 st and $(g+1)$ th column. If $A^{\prime}$ is of rank $m-1$, then some row of $A^{\prime}$ must be a linear combination of the others. Then the required integer $i_{1}$ can be taken to be the smallest index of such row of $A^{\prime}$. However, if $A^{\prime}$ is of rank $m$ then one can take $i_{1}=1$.

Repeating this process $g-k$ times completes the proof.

2.5. TheOREM. Let $k$ and $g$ be integers with $1 \leq k \leq g, m=k+g, G=\left(\mathbf{Z}_{p}\right)^{m}$,

$$
\xi: M^{*}(g, G) \rightarrow H_{2}(G)
$$

the symplectic invariant, defined above, and $\mathrm{v} \in H_{2}(G)$ any vector. Then $\mathrm{v} \in \operatorname{Im}(\xi)$ if and only if there exists an increasing sequence $l$ of $g-k$ natural numbers not exceeding $m$ such that $d\left(\mathbf{v}^{l}\right) \neq 0(\bmod p)$. Note that if $k=g$, then $I$ is empty, and in this case $\mathbf{v}^{l}$ is taken to be v.

Proof. The necessity follows from Corollary 2.3. and Proposition 2.4. The proof of the sufficiency is divided into 2 cases.

Case (1): $(k=g)$. We use induction on $g$.

For $g=1$, and $v \in H_{2}(G)-\{0\}$, let

$$
A=\left[\begin{array}{cc}
1 & 0 \\
0 & v_{12}
\end{array}\right] .
$$

Then $A \in M(g, G)$ and $\xi(A)=\mathbf{v}$.

Assume the result is true for all natural numbers less than $g$, where $g>1$. The fact that $d(v) \neq 0(\bmod p)$ implies that there exists an integer $r$ such that $2 \leq r \leq 2 g$ and $d\left(\mathbf{v}^{r}\right) \neq 0(\bmod p)$. Without loss of generality we may assume that $r=2$. By the induction 
hypothesis, there exists a matrix $B \in M\left(g-1, G /\left(\mathbf{Z}_{p}\right)^{2}\right)$ such that $\xi(B)=\mathbf{v}^{2}$. Let

$$
C=\left[\begin{array}{cccccccc}
1 & 0 & \ldots & 0 & 0 & 0 & \ldots & 0 \\
0 & b_{11} & \ldots & b_{1 g-1} & v_{23} & b_{1 g} & \ldots & b_{12(g-1)} \\
& & \vdots & & & & \vdots & \\
0 & b_{2(g-1) 1} & \ldots & b_{2(g-1) g-1} & v_{22 g} & b_{2(g-1) g} & \ldots & b_{2(g-1) 2(g-1)}
\end{array}\right]
$$

and consider the linear system of equations

$$
C J_{2 g} \mathrm{x}=\left(v_{12} \ldots v_{12 g}\right)^{\mathrm{t}}
$$

Since $\operatorname{rank}(C)=2 g-1$, then a solution exists. If $\mathbf{u}=\left(x_{1} \ldots x_{g} x_{g+1} \ldots x_{2 g}\right)$, and $A$ is the $2 g \times 2 g$ matrix whose 1 st row is $\mathbf{u}$ and the rest are those of $C$, then $A \in M(g, G)$ and $\xi(A)=\mathbf{v}$.

The proof in this case, then, follows by induction.

Case (2): $(k<g)$. Let $\mathbf{v} \in H_{2}(G)$, and let $I$ be an increasing sequence of $g-k$ natural numbers not exceeding $m$ such that $d\left(\mathbf{v}^{\prime}\right) \neq 0(\bmod p)$. Without loss of generality, assume that $l=(1,2, \ldots, g-k)$. Since $\mathbf{v}^{l} \in H_{2}\left(G /\left(\mathbf{Z}_{p}\right)^{g-k}\right)$, then, by case $(1)$, there exists a $2 k \times 2 k$ matrix $B \in M\left(k, G /\left(\mathbf{Z}_{p}\right)^{g-k}\right)$ such that $\xi(B)=\mathbf{v}^{I}$. If

$$
A=\left[\begin{array}{cccccccccccccc}
1 & 0 & \ldots & 0 & 0 & \ldots & 0 & 0 & 0 & \ldots & 0 & 0 & \ldots & 0 \\
0 & 1 & \ldots & 0 & 0 & \ldots & 0 & v_{12} & 0 & \ldots & 0 & 0 & \ldots & 0 \\
0 & 0 & \ldots & 0 & 0 & \ldots & 0 & v_{13} & v_{23} & \ldots & 0 & 0 & \ldots & 0 \\
& & \vdots & & & \vdots & & & & \vdots & & & \vdots & \\
0 & 0 & \ldots & 1 & 0 & \ldots & 0 & v_{1 g-k} & v_{2 g-k} & \ldots & 0 & 0 & \ldots & 0 \\
0 & 0 & \ldots & 0 & b_{11} & \ldots & b_{1 k} & v_{1 g-k+1} & v_{2 g-k+1} & \ldots & v_{g-k g-k+1} & b_{1 k+1} & \ldots & b_{12 k} \\
0 & 0 & \ldots & 0 & b_{2 k 1} & \ldots & b_{2 k k} & v_{1 g+k} & v_{2 g+k} & \ldots & v_{g-k g+k} & b_{2 k k+1} & \ldots & b_{2 k 2 k}
\end{array}\right]
$$

then $A \in M(g, G)$ and $\xi(A)=\mathbf{v}$.

As an example, this means that if $k=1$ then for any $g, \operatorname{Im}(\xi)=H_{2}(G)-\{0\}$. And for $k=g=2, \mathrm{v}=\left(v_{12}, v_{13}, v_{14}, v_{23}, v_{24}, v_{34}\right) \in \operatorname{Im}(\xi)$ if and only if

$$
v_{12} v_{34}-v_{13} v_{24}+v_{14} v_{23} \neq 0 \quad(\bmod p) \text {. }
$$

\section{REFERENCES}

1. E. Artin, Geometric algebra (Interscience, 1957).

2. A. L. Edmonds, Surface symmetry I, Michigan Math. J. 29 (1982), 171-183.

3. A. L. Edmonds, Surface symmetry II, Michigan Math. J. 30 (1983), 143-154:

4. S. A. Jassim, Finite abelian surface coverings, Glasgow Math. J. 25 (1984), 207-218.

5. S. A. Jassim, Classifications of covering spaces (Ph.D. thesis, University College of Swansea, Wales, 1980).

6. W. B. R. Lickorish, A finite set of generators for the homeotopy group of a 2-manifold, Proc. Cambridge Philos. Soc. 60 (1964), 769-778.

7. C. Livingston, Inequivalent, bordant group actions on a surface, Math. Proc. Cambridge Philos. Soc. 99 (1986), 233-238.

8. P. A. Smith, Abelian actions on 2-manifolds, Michigan Math. J. 14 (1967), 257-275.

9. K. Yokoyama, Classification of periodic maps on compact surfaces: I, Tokyo J. Math. 6 (1983), 75-94. 
10. K. Yokoyama, Classification of periodic maps on compact surfaces: II, Tokyo J. Math. 7 (1984), 249-285.

11. B. Zimmermann, Surfaces and the second homology of a group, Monotsh. Math. 104 (1987), 247-253.

Department of Computer Science

UNIVERSITY OF BUCKINGHAM

U.K. 